\section{Iniquidade na assistência à gestação e ao parto em município do semiárido brasileiro}

\section{Inequality in health care for women during gestation and delivery in a municipality in the semi-arid region of Brazil}

Patrícia S. Mano 1

Juraci A. Cesar 2

David A. González-Chica 3

Nelson A. Neumann 4

\begin{abstract}
Objectives: to evaluate inequality in health care during gestation and delivery for women with children under five years of age resident in the Municipality of Caracol, in the Brazilian State of Piauí.

Methods: a household questionnaire was applied to a cross-sectional systematic sample to collect data on the demographic characteristics of mothers and children, health care during pregnancy and deliver and socio-economic status. A tercile score was created using the principal component technique. The associations between this score and variables relating to health care for women during pregnancy and childbirth were evaluated using the chi-square and Kruskal-Wallis test.

Results: all the 405 children covered by the study came from families with an income of less than one minimum wage per month; $65 \%$ lived in rural areas. Mothers belonging to the highest socio-economic tercile had a larger number of pre-natal consults; they had the largest number of urine tests, hemograms, cytopathological tests of the cervix and ultrasound, and their babies were delivered by a doctor with a higher frequency of caesarian sections.

Conclusions: even among the poorest sectors of society there are enormous health inequalities. It is imperative that the factors determining this poverty be addressed with more aggressive social programs and priority given to caring for the poorest of the poor.
\end{abstract}

Key words Maternal and child health, Prenatal care, Health inequalities, Comprehensive health care
1,2 Divisão de População \& Saúde. Universidade Federal do Rio Grande. Rua General Osório, s.n. $4^{\circ}$ andar. Centro. Campus Saúde Área Acadêmica Prof. Newton Azevedo. Rio Grande, RS, Brasil. CEP: 96.201-900. E-mail: juraci.cesar@gmail.com

3 Departamento de Nutrição. Universidade Federal de Santa Catarina. Florianópolis, SC, Brasil.

4 Coordenação Nacional da Pastoral da Criança. Curitiba, PR, Brasil.

\section{Resumo}

Objetivos: avaliar a iniquidade na assistência à gestação e ao parto para menores de cinco anos residentes no município de Caracol, Piaui, Brasil.

Métodos: usando delineamento transversal, amostragem sistemática e aplicação de questionário domiciliar foram coletadas informações sobre características demográficas de mães e crianças, assistência à gestação e ao parto e nível socioeconômico. Por meio da técnica de componentes principais, criou-se um escore em tercis. As associações deste escore com as variáveis relativas à assistência à gestação e ao parto foram avaliadas pelos testes qui-quadrado e Kruskal-Wallis.

Resultados: todas as 405 crianças incluidas no estudo eram provenientes de famílias com renda inferior a um salário mínimo mensal; $65 \%$ residiam em área rural. Mães pertencentes ao maior tercil socioeconômico realizaram um maior número de consultas de pré-natal; realizaram maior número de exames de urina, hemograma, citopatológico de colo uterino e ultrassonografia, tiveram o parto realizado por médico e foram mais frequentemente submetidas à cesariana.

Conclusões: mesmo entre os mais pobres, há enormes iniquidades em saúde. Combater os determinantes desta pobreza com programas sociais mais agressivos é imperativo, assim como priorizar o atendimento aos mais pobres dentre os pobres.

Palavras-chave Saúde materno-infantil, Assistência pré-natal, Desigualdades em saúde, Assistência integral à saúde da criança 


\section{Introdução}

Iniquidade é a existência de diferenças desnecessárias, evitáveis, igualmente abusivas e injustas. ${ }^{1}$ Resulta da distribuição desigual de poder, prestígio e recursos entre grupos sociais. ${ }^{2}$ Em saúde, manifestase mais comumente, mostrando menor cobertura e/ou pior qualidade dos serviços oferecidos aos mais pobres.

Diversos estudos têm sido realizados no Brasil buscando avaliar iniquidades em saúde maternoinfantil. ${ }^{3-5}$ Pelo menos $90 \%$ das gestantes brasileiras iniciam o pré-natal e cerca de $80 \%$ delas encontramse adequadamente imunizadas contra tétano neonatal. Praticamente todas as crianças nascem em hospital, com a grande maioria delas sendo atendidas por médicos; de cada mil nascidos vivos, cerca 20 vão ainda a óbito até o final do primeiro ano de vida. 6 No entanto, este cenário esconde enormes iniquidades em diferentes níveis. Por exemplo, 10\% das crianças da Região Norte do Brasil nascem fora do ambiente hospitalar contra $1 \%$ na Região Sul; no Estado de Sergipe, $48 \%$ das mães realizam sete ou mais consultas de pré-natal contra apenas $23 \%$ no Estado do Maranhão; 6 na cidade de Pelotas, $73 \%$ das gestantes iniciaram o pré-natal no primeiro trimestre, 7 enquanto em Rio Grande, um município vizinho, isto ocorreu para somente $66 \%$ das riograndinas. ${ }^{8}$ Evidencia-se, portanto, a existência de iniquidades.

Estudos sobre iniquidade em saúde concentramse em avaliar (e denunciar) sua existência entre ricos e pobres, regiões ou municípios, mas raramente avaliam-na entre os mais pobres. Isto porque os estudos partem do pressuposto de que os pobres são iguais e que, portanto, são analogamente afetados por este tipo de problema, o que pode não ser verdade. ${ }^{9}$

No presente artigo busca-se avaliar a existência de iniquidade na assistência à gestação e ao parto no município de Caracol, Estado do Piauí, um dos mais pobres do Brasil. Para tanto, incluiu apenas crianças menores de cinco anos cujas famílias possuíam renda mensal inferior a um salário mínimo.

\section{Métodos}

O presente estudo faz parte de um trabalho mais amplo, realizado em 2002 e repetido em 2005, que tinha por objetivo avaliar indicadores básicos de saúde materno-infantil em 18 áreas de nove municípios entre os 36 mais pobres das Regiões Norte e Nordeste do Brasil, conforme classificação do Fundo das Nações Unidas para a Infância e Adolescência
(Unicef) e Pastoral da Criança. ${ }^{10}$

O município de Caracol situa-se na região sul do Estado do Piauí, a 470 km da sua capital. Em 2005, sua população era composta por cerca de 9 mil habitantes. ${ }^{11}$ Possuía duas unidades básicas de saúde e um centro de saúde. O Produto Interno Bruto (PIB) per capita era de $\mathrm{R} \$ 1.954,00.11$ O Índice de Desenvolvimento Humano (IDH) era de 0,59 e a taxa de mortalidade infantil de 29/1000 nascidos vivos. 12

A população-alvo deste estudo foi constituída por todas as crianças menores de cinco anos $(0$ a 59 meses) residentes em metade dos domicílios deste município entre os meses de março e setembro de 2005 e cuja renda familiar no mês anterior a entrevista fosse inferior a um salário mínimo.

Considerando margem de erro de 4,5 pontos percentuais, prevalência de renda familiar mensal inferior a um salário mínimo entre famílias com crianças menores de cinco anos de $70 \%$ no município e $5 \%$ para eventuais perdas, o presente estudo deveria incluir pelo menos 410 mães. O delineamento usado neste estudo foi do tipo transversal.

Empregou-se amostragem sistemática a partir de mapeamento e numeração das áreas, seguido de sorteio de um ponto inicial e sorteio aleatório do primeiro domicílio, sempre em sentido horário. Assim, visitava-se um domicílio e outro não. Isto foi feito em todas as comunidades, urbanas e rurais, até completar todos os domicílios do município.

Questionário padronizado, pré-testado, foi aplicado por seis entrevistadores previamente treinados a todas as mães ou responsáveis pela guarda de menores de cinco anos. As principais informações coletadas foram características demográficas e socioeconômicas, condições de habitação e saneamento, assistência à gestação e ao parto, padrão de morbidade, de amamentação e dieta e o uso de serviços de saúde. Embora a maioria das variáveis seja auto-explicativa, algumas merecem esclarecimentos adicionais: cor da pele (classificada pelo entrevistador em branca, parda/morena e preta); renda familiar mensal (valor recebido por todos os moradores do domicílio no mês anterior à entrevista); imunização antitetânica; total de doses recebidas em gravidezes anteriores e na atual.

Os questionários eram codificados, revisados e duplamente digitados, sendo posteriormente, comparados e corrigidos. Todas estas etapas foram realizadas usando-se o programa Epi-Info, versão 6.04. Os dados foram analisados por meio do programa Stata, versão 9.2. O controle de qualidade deste estudo foi realizado revisando os questionários 
e repetindo parcialmente cerca de $10 \%$ das entrevistas.

Para analisar iniquidades foi construído um escore de nível socioeconômico das famílias estudadas que considerou variáveis socioeconômicas (escolaridade materna e renda familiar), condições de moradia (posse de eletrodomésticos, tipo de construção, tipo de piso, número de compartimentos usados para dormir, acesso à rede de esgotos e água tratada, disponibilidade de sanitário) e acesso aos serviços de saúde. Para definir quais informações constituiriam o escore, foi empregada análise de componentes principais, ${ }^{13}$ que permitiu reduzir o conjunto de variáveis a um número menor de componentes que explicassem a maior variância dos

\section{Tabela 1}

Características demográficas e socioeconômicas das mães de menores de cinco anos residentes no município de Caracol, PI, 2005.

\begin{tabular}{|c|c|c|}
\hline Variável & $\mathbf{N}$ & $\%$ \\
\hline \multicolumn{3}{|c|}{ Área em que vive } \\
\hline Urbana & 143 & 35,3 \\
\hline Rural & 262 & 64,7 \\
\hline \multicolumn{3}{|c|}{ Cor da pele da mãe } \\
\hline Branca & 29 & 7,2 \\
\hline Parda/Mulata & 274 & 67,6 \\
\hline Preta & 102 & 25,2 \\
\hline \multicolumn{3}{|l|}{ Idade (anos) } \\
\hline$<20$ & 39 & 9,6 \\
\hline 20 a 29 & 300 & 74,1 \\
\hline 30 а 39 & 52 & 12,8 \\
\hline 40 ou mais & 14 & 3,5 \\
\hline $\bar{X} \pm D P$ & $25,3 \pm 6,0$ & \\
\hline \multicolumn{3}{|c|}{ Escolaridade da mãe (anos) } \\
\hline Nenhum & 77 & 19,0 \\
\hline 1 a 4 & 165 & 40,7 \\
\hline 5 a 8 & 131 & 32,3 \\
\hline 9 ou mais & 32 & 7,9 \\
\hline $\bar{X} \pm D P$ & $3,8 \pm 2,8$ & \\
\hline \multirow{2}{*}{\multicolumn{3}{|c|}{$\begin{array}{l}\text { Renda familiar mensal } \\
\text { (salários mínimos) }\end{array}$}} \\
\hline & & \\
\hline 0 a 0,24 & 149 & 36,8 \\
\hline 0,25 a 0,49 & 109 & 26,9 \\
\hline 0,50 a 0,74 & 69 & 17,0 \\
\hline 0,75 a 0,99 & 78 & 19,3 \\
\hline $\bar{X} \pm D P$ & $0,42 \pm 0,28$ & \\
\hline Mediana & 0,38 & \\
\hline \multicolumn{3}{|c|}{ Moradores por domicílio } \\
\hline $\bar{X} \pm D P$ & $4,8 \pm 1,7$ & \\
\hline Total & 405 & 100,0 \\
\hline
\end{tabular}

dados analisados. O primeiro componente desta análise apresentou um eigenvalue de 1,81 (explicando $30 \%$ da variabilidade dos dados), a partir do qual foi gerado um escore contínuo que representa o nível socioeconômico das famílias. As variáveis mantidas para a criação do escore foram posse de eletrodomésticos, energia elétrica, tipo de sanitário, água encanada, escolaridade materna e distância ao posto de saúde, com pesos no escore entre 0,35 e 0,51. O escore socioeconômico foi posteriormente dividido em tercis, para uso nas análises, considerando que o tercil inferior representa as famílias mais pobres do município.

A iniquidade foi avaliada por meio da categorização do escore em tercis de pobreza, do mais pobre ao menos pobre, recebendo a denominação de escore de pobreza. O escore foi cruzado com cada uma das variáveis relativas ao pré-natal. As associações foram avaliadas por meio do teste quiquadrado de Pearson para proporções enquanto que, para desfechos contínuos, empregou-se o teste Kruskal-Wallis, adotando-se o erro alfa de, no máximo, 0,05.14

O protocolo de pesquisa foi submetido e aprovado pelo Comitê de Ética em Pesquisa na Área da Saúde (CEPAS) da Universidade Federal do Rio Grande.

\section{Resultados}

Visitando 50\% dos domicílios de Caracol de forma sistemática, este estudo identificou 416 crianças menores de cinco anos cuja renda familiar mensal foi inferior a um salário mínimo. As perdas totalizaram 2,6\%. Assim, o denominador deste estudo foi constituído por 405 crianças.

A Tabela 1 mostra que cerca de dois terços das mães incluídas no estudo residiam em área rural, possuíam cor da pele parda/mulata, cerca de três quartos tinham entre 20 e 29 anos de idade, aproximadamente $20 \%$ não eram alfabetizadas, $65 \%$ possuíam renda familiar de, no máximo, meio salário mínimo mensal; em média residiam, cinco pessoas por domicílio. Isto resultava em uma renda per capita de um dólar por pessoa/dia (1 U\$ = R $\$ 1,60)$.

$\mathrm{Na}$ Tabela 2 verifica-se que a quase totalidade das moradias era construída de tijolos/bloco, embora a argamassa fosse de barro; metade dos domicílios possuía piso de terra batida, somente $2 \%$ tinham água encanada dentro de casa e $65 \%$ usavam água proveniente de poço, cisterna, açude ou rio; $73 \%$ dos domicílios não possuíam qualquer tipo de sanitário, $25 \%$ não possuíam energia elétrica; rádio e televisão eram os eletrodomésticos mais comumente encon- 
trados nos domicílios com $60 \%$ e $44 \%$, respectivamente. Nove em cada dez domicílios usavam fogão a lenha ou carvão.

A Tabela 3 trata da assistência à gestação e ao parto de acordo com o tercil de pobreza. Mães pertencentes ao tercil menos pobre mostraram, de forma sistemática, vantagem para diversos destes indicadores em relação àquelas do pior tercil de pobreza, ou seja, as mais pobres dentre os pobres. Mães do tercil menos pobre realizaram um maior número de consultas durante o pré-natal, tiveram a altura uterina e o peso mais frequentemente verificados, realizaram em maior proporção, exames de urina e hemograma e ultrassonografia obstétrica; seus filhos nasceram mais comumente em maternidade e seus partos foram mais frequentemente realizados por médicos.

Por fim, a Figura 1 mostra a ocorrência de cesrianas entre todas as mães conforme o tercil de renda. A taxa de cesarianas entre todas as mães foi de $16 \%$, variando de $6 \%$ no tercil mais pobre a $27 \%$ no tercil menos pobre. Isto mostra que o risco de uma mãe pertencente ao tercil menos pobre ter filho por cesariana é 4,5 vezes maior em relação aquelas do tercil mais pobre $(27 / 6=4,5)$.

Tabela 2

Condições de moradia para mães de menores de cinco anos residentes no município de Caracol, PI, 2005.

\begin{tabular}{|c|c|c|}
\hline Variável & $\mathbf{N}$ & $\%$ \\
\hline \multicolumn{3}{|l|}{ Tipo de construção da moradia } \\
\hline Tijolo/Bloco & 398 & 98,3 \\
\hline Taipa & 06 & 1,5 \\
\hline Madeira & 00 & 0,0 \\
\hline Outro & 01 & 0,2 \\
\hline \multicolumn{3}{|l|}{ Tipo de piso } \\
\hline Cimento & 200 & 49,6 \\
\hline Chão/Terra batida & 190 & 46,9 \\
\hline Outro & 15 & 3,5 \\
\hline \multicolumn{3}{|l|}{ Vive em casa } \\
\hline Própria & 312 & 77,0 \\
\hline Emprestada/Cedida & 91 & 22,5 \\
\hline Alugada & 02 & 0,5 \\
\hline \multicolumn{3}{|l|}{ Água encanada } \\
\hline Dentro de casa & 09 & 2,2 \\
\hline No pátio/terreno & 63 & 15,6 \\
\hline Não tem & 333 & 82,2 \\
\hline \multicolumn{3}{|l|}{ Origem da água usada para beber } \\
\hline Rede pública & 04 & 1,0 \\
\hline Cisterna/Poço & 172 & 42,5 \\
\hline Rio/Lago/Lagoa/Açude & 93 & 23,0 \\
\hline Outros & 136 & 33,5 \\
\hline \multicolumn{3}{|l|}{ Tipo de privada } \\
\hline Sanitário com descarga & 18 & 4,4 \\
\hline Sanitário sem descarga & 70 & 17,3 \\
\hline Casinha/Fossa negra & 21 & 5,2 \\
\hline Não tem & 296 & 73,1 \\
\hline Domicílios com energia elétrica & 306 & 75,6 \\
\hline \multicolumn{3}{|c|}{ Disponibilidade de eletrodomésticos } \\
\hline Rádio & 245 & 60,5 \\
\hline Televisão & 177 & 43,7 \\
\hline Geladeira & 79 & 19,5 \\
\hline Fogão a gás & 167 & 41,2 \\
\hline Fogão a lenha/carvão & 359 & 88,6 \\
\hline Total & 405 & 100,0 \\
\hline
\end{tabular}


Tabela 3

Assistência à gestação e ao parto para mães de menores de cinco anos residentes no município de Caracol, Pl, 2005.

\begin{tabular}{|c|c|c|c|c|c|}
\hline \multirow[t]{3}{*}{ Variável } & \multicolumn{3}{|c|}{ Tercil de pobreza* } & \multirow[t]{2}{*}{ Total $(n=405)$} & \multirow[t]{3}{*}{$p^{* *}$} \\
\hline & $1^{\circ}(n=135)$ & $2^{\circ}(n=135)$ & $3^{\circ}(n=135)$ & & \\
\hline & $\%$ & $\%$ & $\%$ & $\%$ & \\
\hline Iniciaram as consultas de pré-natal & 90,3 & 91,8 & 96,3 & 92,8 & 0,14 \\
\hline Iniciaram o pré-natal no primeiro trimestre & 83,2 & 84,0 & 84,2 & 83,8 & 0,93 \\
\hline \multicolumn{6}{|l|}{ Número de consultas de pré-natal } \\
\hline Seis ou mais & 59,0 & 56,1 & 64,7 & 59,9 & 0,34 \\
\hline Nove ou mais & 14,2 & 14,4 & 25,6 & 18,0 & 0,02 \\
\hline $\bar{X} \pm D P$ & $5,5 \pm 2,7$ & $5,4 \pm 2,8$ & $6,7 \pm 3,4$ & $5,9 \pm 3,0$ & 0,007 \\
\hline Imunização contra tétano neonatal & 48,8 & 51,5 & 49,2 & 49,9 & 0,89 \\
\hline \multicolumn{6}{|l|}{ Verificação da (o) } \\
\hline Pressão arterial & 96,7 & 95,9 & 98,4 & 97,0 & 0,48 \\
\hline Peso da gestante & 97,5 & 91,1 & 98,4 & 95,7 & 0,008 \\
\hline Batimentos cardíacos do feto & 97,5 & 98,4 & 97,7 & 97,8 & 0,84 \\
\hline Altura uterina & 74,4 & 85,4 & 88,9 & 83,0 & 0,007 \\
\hline Edema de membros inferiores & 70,2 & 68,3 & 70,3 & 69,6 & 0,92 \\
\hline \multicolumn{6}{|l|}{ Se durante as consultas de pré-natal } \\
\hline Fez exame ginecológico & 22,3 & 26,0 & 23,6 & 24,0 & 0,78 \\
\hline Teve as mamas examinadas & 28,1 & 35,0 & 33,6 & 32,6 & 0,47 \\
\hline Recebeu sulfato ferroso & 92,6 & 97,6 & 95,3 & 95,2 & 0,19 \\
\hline \multicolumn{6}{|l|}{ Tipo de exame realizado } \\
\hline Urina & 19,0 & 18,7 & 34,6 & 24,3 & 0,003 \\
\hline Sangue & 23,1 & 21,3 & 33,9 & 26,2 & 0,05 \\
\hline Exame para câncer no útero & 14,0 & 5,7 & 12,6 & 10,8 & 0,07 \\
\hline Ultrassonografia obstétrica & 47,9 & 56,1 & 71,9 & 58,9 & $<0,001$ \\
\hline Local de nascimento & & & & & 0,008 \\
\hline Hospital/Maternidade & 88,2 & 83,7 & 94,1 & 88,6 & \\
\hline No próprio domicílio & 11,8 & 16,3 & 5,9 & 11,4 & \\
\hline Quem fez o parto & & & & & $<0,001$ \\
\hline Médico & 38,5 & 45,2 & 68,1 & 50,6 & \\
\hline Enfermeira & 38,5 & 29,6 & 23,0 & 30,4 & \\
\hline Parteira & 20,0 & 18,5 & 8,1 & 15,6 & \\
\hline Ninguém & 3,0 & 6,7 & 0,7 & 3,5 & \\
\hline
\end{tabular}

*As variáveis usadas na construção do escore por meio da análise de componentes principais foram posse de eletrodomésticos, energia elétrica, tipo de sanitário, água encanada, escolaridade materna e distância ao posto de saúde, com pesos no escore entre 0,35 e $0,51.1^{\circ}$ tercil = pior situação; $3^{\circ}$ tercil = melhor situação. O escore socioeconômico foi posteriormente dividido em tercis para usar nas análises considerando que o pior tercil representa as famílias mais pobres do município; ** Teste qui-quadrado, exceto para consultas (KruskalWallis). 


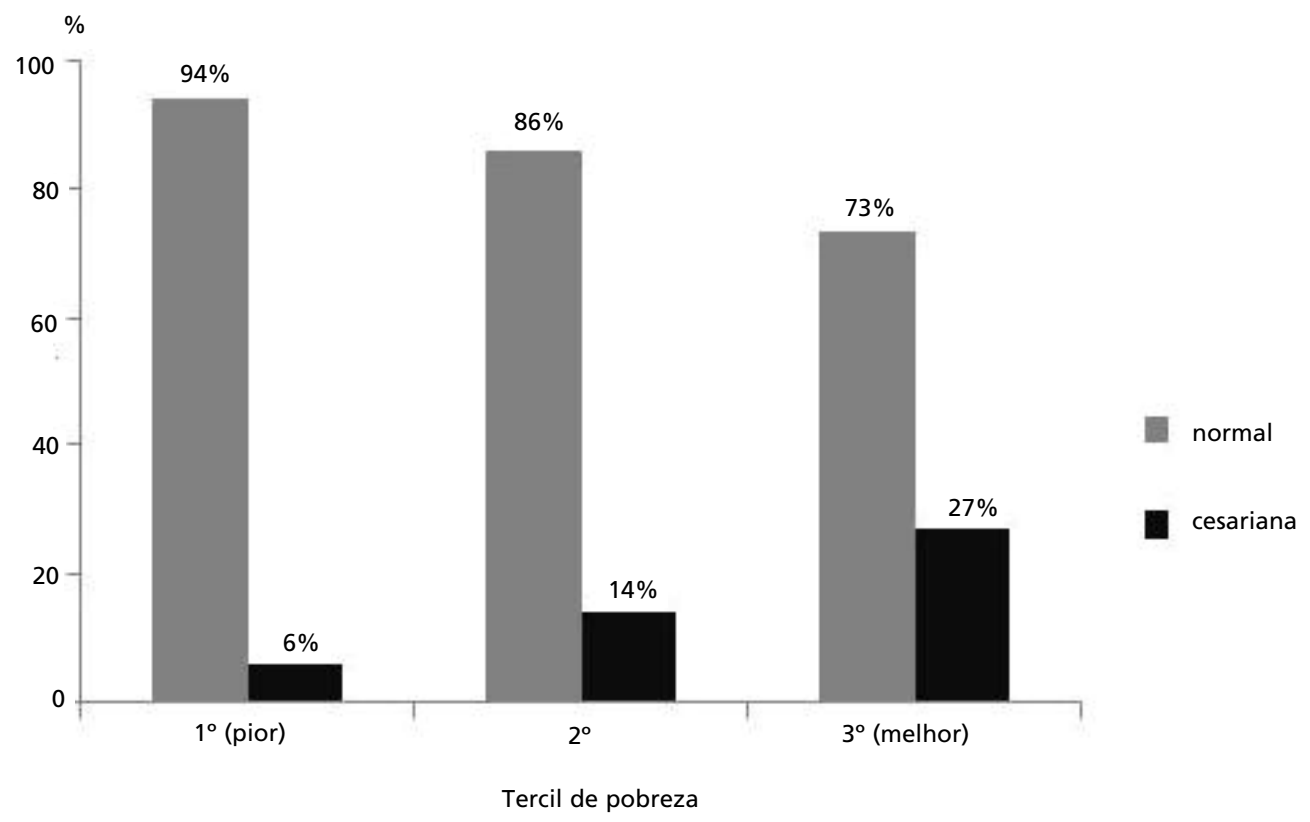

$p<0,001 ; n=405$.

\section{Discussão}

Este estudo revelou o extremo grau de pobreza em que sobrevivem as famílias, com indicadores muito aquém das demais regiões do país.6,15 Esta pobreza é demonstrada pela baixa escolaridade das mães, baixo poder aquisitivo das famílias, elevada aglomeração domiciliar, péssimas condições de habitação e saneamento e inadequada assistência à gestação e ao parto. Evidenciou ainda que mesmo entre os pobres existe iniquidade. Isto porque mães pertencentes ao melhor tercil de pobreza mostraram cobertura sistematicamente maior para diversos indicadores em relação às mães do pior tercil de pobreza.

$\mathrm{O}$ estudo das iniquidades em assistência prénatal tem sido analisado a partir da variável renda familiar.3,16-18 Neste estudo, a renda, apenas, não foi suficiente para mostrar estas diferenças. Tampouco o foi a variável escolaridade, um dos mais importantes determinantes da saúde infantil.18,19 Isto provavelmente aconteceu por serem muito pobres e uma única variável não se mostrou capaz de captar tais diferenciais, daí a razão do uso da análise de componentes principais. Logo, a comparação com outros estudos fica prejudicada e deve ser levado em consideração ao interpretar estes dados. Por fim, vale lembrar que se trata de um delineamento trans- versal, o que implica mensuração da exposição e do desfecho de forma simultânea e, por conseguinte, a impossibilidade de estabelecer qualquer tipo de relação de causalidade entre os eventos estudados.

Mães pertencentes ao tercil menos pobre realizaram, em média, ao longo de todo o pré-natal, 1,2 consultas a mais em relação àquelas do tercil mais pobre. Em Campinas, SP, mulheres pertencentes ao grupo com renda per capita inferior a um salário mínimo iniciaram mais tardiamente as consultas de pré-natal e realizaram um menor número de consultas; 3 em Pelotas, RS, 38\% das mulheres pertencentes ao quintil de renda mais baixo tinham realizado pré-natal inadequado, enquanto que no quintil de maior renda esta proporção era de apenas 16\%; 20 em Criciúma, SC, enquanto $74 \%$ e $67 \%$ das gestantes pertencentes ao pior quartil de renda iniciaram as consultas de pré-natal no primeiro trimestre e realizaram seis ou mais consultas, isto ocorreu, respectivamente, para $88 \%$ e $84 \%$ daquelas pertencentes ao melhor quartil de renda; 17 em Rio Grande, RS, cerca de dois terços das gestantes mais pobres (primeiro quartil) iniciaram o pré-natal e realizaram seis ou mais consultas enquanto entre as mais ricas (quarto quartil) o percentual foi de cerca de três quartos. 21

Para pelo menos dois dos cinco exames clínicos 
e quatro dos seis testes laboratoriais minimamente recomendados durante o pré-natal, mães pertencentes ao melhor tercil apresentaram coberturas significativamente maiores em relação ao tercil mais pobre. Isto também foi observado de forma muito mais acentuada em praticamente todos os exames clínicos e testes laboratoriais nas localidades de Criciúma (SC), ${ }^{17}$ Rio Grande (RS), ${ }^{21}$ Embu (SP) 22 e Campinas (SP). ${ }^{3}$ A realização de, pelo menos, seis consultas de pré-natal e de todos os exames clínicos e testes laboratoriais básicos poderia reduzir em até $10 \%$ os índices de mortalidade infantil. 15

Em relação ao profissional que fez o parto, as iniquidades mostram-se mais evidentes ainda. Enquanto sete em cada dez partos do tercil menos pobre foram atendidas por médicos, o mesmo ocorreu para apenas quatro em cada dez do tercil mais pobre. Ao mesmo tempo, a realização de parto por parteira foi 2,5 vezes maior entre aquelas do pior tercil de pobreza em relação às mães do melhor tercil.

A Pesquisa Nacional em Demografia e Saúde (PNDS) de 2006 mostrou que 98\% das mães do quintil superior, de bens, tiveram o parto atendido por médico comparadas com $52 \%$ no quintil inferior. $23 \mathrm{O}$ estudo das coortes de nascimento de Pelotas mostrou que a proporção de mães das famílias de menor renda cujos partos foram atendidos por médicos aumentou de $69 \%$ em 1982 para $86 \%$ em 2004. Entre as mães das famílias mais ricas, passou de $93 \%$ em 1982 para $98 \%$ em 2004.7

A baixa ocorrência de cesariana em ambos os municípios, embora pareça próximo do índice recomendado pela Organização Mundial da Saúde, cerca de $15 \%, 24$ esconde importantes iniquidades. 25 A proporção de gestantes do melhor tercil submetida à cesariana foi 4,5 vezes maior em relação àquelas do pior tercil. Esta mesma tendência foi observada entre 1982 e 2004 em Pelotas, onde a prevalência de

\section{Referências}

1. Whitehead M. The concepts and principles of equity and health. Int J Health Serv. 1992; 22: 429-45.

2. Marmot M. Achieving health equity: from root causes to fair outcomes. Lancet. 2007; 370 (9593): 1153-63.

3. Almeida SD, Barros MB. Equity and access to health care for pregnant women in Campinas (SP), Brazil. Rev Panam Salud Publica. 2005; 17: 15-25.

4. Coutinho T, Teixeira MTB, Dain S, Sayd JD, Coutinho LM Adequação do processo de assistência pré-natal entre usuárias do Sistema Único de Saúde em Juiz de Fora - MG. Rev Bras Ginecol Obstet. 2003; 25: 717-24.

5. Trevisan MR, Lorenzi DRS, Araújo NM, Ésber K. Perfil da Assistência pré-natal entre usuárias do Sistema Único de cesarianas para mulheres com renda familiar superior a dez salários mínimos mensais aumentou de 47\% em 1982 para 79\% em 2004.7 Na PNDS, o índice global foi de $36 \%$, variando de $16 \%$ no quintil inferior a $67 \%$ no quintil superior. ${ }^{23}$

A assistência à gestação e ao parto mostrou que, mesmo entre os mais pobres, há iniquidades. Estudo realizado em áreas rurais da Tanzânia, também entre os mais pobres, mostrou que o uso de serviços curativos de saúde foi significativamente menor entre crianças pertencentes ao pior quintil de pobreza. ${ }^{9}$

Os indicadores de assistência à gestação e ao parto observados em 2005 em Caracol são semelhantes ao obtido entre 1982-1984 para recémnascidos de Pelotas, RS, cujas famílias possuíam também renda familiar inferior a um salário mínimo mensal, os mais pobres de Pelotas, RS. $18 \mathrm{O}$ atraso é, portanto, de pelo menos um quarto de século.

Tão importante quanto estudar iniquidades é combater os seus determinantes $\mathrm{e}$ as suas origens. 25,26 É evidente a necessidade de investir nestas populações, e isto implica melhoria nas condições de educação, saúde, emprego, habitação, exploração da terra, moradia, acesso a bens, entre outros. Neste sentido, políticas públicas e programas de governo mais agressivos deveriam ser adotados. Prioridade deveria ser dada aos mais pobres dos pobres. Do contrário, as iniquidades continuarão a existir, mesmo entre os aparentemente iguais como a população incluída neste estudo.

\section{Agradecimentos}

Aos professores Juvenal S. Dias-da-Costa do Departamento de Medicina Social da Universidade Federal de Pelotas e Silvio O. M. Prietsch, da Área Materno-Infantil da Universidade Federal do Rio Grande, pelos comentários e sugestões durante a análise dos dados e redação deste artigo.
Saúde em Caxias do Sul. Rev Bras Ginecol Obstet. 2002; 24: 293-9.

6. Brasil. Ministério da Saúde. Indicadores e Dados Básicos do Brasil. [Acesso em 1 out 2008]. Disponível em: http://tabnet.datasus.gov.br/cgi/idb2007/matriz.htm

7. Cesar JA, Matijasevich A, Santos IS, Barros AJD, Dias-daCosta JS, Barros FC, Victora CG. The use of maternal and child health services in three population-based cohorts in Southern Brazil, 1982-2004. Cad Saúde Pública. 2008; 24 (Suppl 3): S427-36.

8. Cesar JA, Mendoza-Sassi R, Horta BL, Ribeiro PR, D'Avila AC, Santos FM, Martins PB, Brandolt RR. Indicadores básicos de saúde infantil em área urbana no extremo sul do 
Brasil: estimando prevalências e avaliando diferenciais. J Pediatr (Rio J). 2006; 82: 437-44

9. Schellenberg JA, Victora CG, Mushi A, de Savigny D, Schellenberg D, Mshinda H, Bryce J. Inequities among the very poor: health care for children in rural southern Tanzania. Lancet. 2003; 361 (9357): 561-6.

10. Cesar JA, Gonçalves TS, Neumann NA, Oliveira Filho JA, Diziekaniak AC. Child health in poor areas of North and Northeast Brazil: a comparison of areas covered by the Children's Mission and control areas. Cad Saúde Pública. 2005; 21: 1845-55

11. IBGE (Instituto Brasileiro de Geografia e Estística). [Acesso em 27 ago 2008]. Disponível em http://www.ibge.gov.br/home/estatistica/economia/pibmunicipios/2005/tab01.pdf

12. IPEA (Instituto de Pesquisas Econômicas e Aplicadas). [Acesso em 20 ago 2008]. Disponível em: http://www.ipeadata.gov.br/ipeaweb.dll/MenuCtrl?SessionID=582418562\& Mod=SOCIAL\&Lang

13. Filmer D, Pritchett LH. Estimating wealth effects without expenditure data--or tears: an application to educational enrollments in states of India. Demography. 2001; 38: 11532.

14. Kirkwood BR, Sterne JAC. Essentials of medical statistics. 2 ed. London: Blackwell Science Ltd; 2003.

15. Victora CG. Intervenções para reduzir a mortalidade infantil pré-escolar e materna no Brasil. Rev Bras Epidemiol. 2001 4: 3-69.

16. Goldani MZ, Barbieri MA, Silva AA, Bettiol H. Trends in prenatal care use and low birthweight in southeast Brazil. Am J Public Health. 2004; 94: 1366-71.

17. Neumann NA, Tanaka OY, Victora CG, Cesar JA. Qualidade e eqüidade da atenção ao pré-natal e ao parto em Criciúma, Santa Catarina, Sul do Brasil. Rev Bras Epidemiol. 2003; 6: 307-18.

Recebido em 21 de outubro de 2010

Versão final apresentada em 29 de agosto de 2011

Aprovado em 20 de setembro de 2011
18. Victora CG, Barros FC, Vaughan JP. Epidemiologia da desigualdade: um estudo longitudinal de 6.000 crianças brasileiras. São Paulo: Hucitec; 1988

19. WHO (World Health Organization). Tracking progress in maternal, newborn and child survival: Countdown to 2015 the 2008 Report. Geneva; 2008.

20. Halpern R, Barros FC, Victora CG, Tomasi E. Prenatal care in Pelotas, Rio Grande do Sul, Brazil, 1993. Cad Saúde Pública. 1998; 14: 487-92.

21. Gonçalves CV, Cesar JA, Mendoza-Sassi RA. Quality and equity in prenatal care: a population- based study in Southern Brazil. Cad Saúde Pública. 2009; 25: 2507-16.

22. Puccini RF, Pedroso GC, Silva EM, Araújo NS, Silva NN. Prenatal and childbirth care equity in an area in Greater Metropolitan Sao Paulo, 1996. Cad Saúde Pública. 2003; 19: 35-45.

23. Brasil. Ministério da Saúde e Centro Brasileiro de Análise e Planejamento. Pesquisa Nacional de Demografia e Saúde da Criança e da Mulher - PNDS 2006: Dimensões do Processo Reprodutivo e da Saúde da Criança. Série G: Estatística e Informação em Saúde. Brasília, DF; 2009.

24. WHO (World Health Organization). Care in normal birth: a practical guide. Geneva; 1996.

25. Gwatkin DR, Rutstein S, Johnson K, Suliman E, Wagstaff A. Socio economic differences in health, nutrition, and population within developing countries: an overview. Washington: World Bank; 2007.

26. Marmot M. Social determinants of health inequalities. Lancet. 2005; 365 (9464): 1099-104. 